\title{
Editorial
}

Emmanuel J. Favaloro and Giuseppe Lippi

\section{On the complexity of hemostasis and the need for harmonization of test practice}

https://doi.org/10.1515/cclm-2018-0174

Hemostasis = Love. Everyone talks about it, but no one understands it.

Hemostasis, a homeostatic process that is meant to prevent blood loss, is achieved by the interaction of various systems, sometimes opposing (or acting to balance proand antihemostatic forces) [1]. Although hemostasis is sometimes also called "coagulation" by some people, this term actually reflects only a small part of hemostasis. Hemostasis comprises four main "systems", although it is impossible to fully separate any from another. The four systems are "primary hemostasis", "secondary hemostasis", "anticoagulation system" and "fibrinolytic system" $[1,2]$. The "primary hemostasis" system is mostly driven by the interaction of blood platelets, adhesive plasma proteins including von Willebrand factor (VWF) and the (damaged) vessel wall comprising endothelium and subendothelial matrix components. The "secondary hemostasis" system is mostly driven by "sequential" activation of procoagulant proteins (the so-called "coagulation cascade") causing generation of thrombin and eventual conversion of fibrinogen to fibrin (the so-called "clot"). The "anticoagulation system" aims to control the process of "secondary hemostasis", so that it does not progress irrevocably, in which case a thrombosis may occur and lead (for example) to deep vein thrombosis (DVT) or pulmonary embolism (PE). The "fibrinolytic system" acts to further control "secondary hemostasis", and also to dissolve any clot formed to enable tissue repair. A breakdown in any of these systems will predispose to either bleeding or thrombosis, depending on what element of the process has failed.

As mentioned, "coagulation" is only a part of hemostasis, and essentially only reflective of the "secondary hemostasis" system. This system comprises many coagulation proteins, perhaps most well-known being factors VIII (FVIII) and IX (FIX), the deficiency of which will, respectively, cause hemophilia $A$ and $B$, thus reflecting the most common inherited bleeding disorders related to secondary hemostasis [3]. The term "coagulation" (or "coag”) laboratory usually refers to laboratories that are capable of performing (a limited number of) "routine" tests of hemostasis, as listed in Table 1.

The four hemostasis systems mentioned are not independent of each other, and all processes intertwine. For example, although VWF is primarily involved in "primary hemostasis", because it binds to, and protects, and also delivers FVIII to sites of vascular damage, it is also integral to the success of "secondary hemostasis". This is why some patients with deficiency or defect in VWF, thereby suffering von Willebrand disease (VWD), may experience bleeding considered due to defects in "primary hemostasis" as well as "secondary hemostasis" [4]. Similarly, fibrinogen is primarily involved in "secondary hemostasis", generating the clot after conversion to fibrin. However, fibrinogen can also bind to activated platelets and, thus, contribute to "primary hemostasis".

The modern hemostasis laboratory has an arsenal of tests it can perform for investigation of hemostasis (Table 1). With these tests, laboratories can help diagnose bleeding disorders such as hemophilia or VWD, explain the occurrence of familial thrombophilia (e.g. deficiency in proteins of the anticoagulant system) or manage patients under therapy (e.g. patients being treated for a DVT with vitamin $\mathrm{K}$ antagonist [VKA] therapy). However, the ability of laboratories to help clinicians diagnose hemostatic "disease" or better manage patients is compromised by lack of standardization and harmonization. Different laboratories may have the appearance of having the "same tests" in their repertoire, but given the high variety of test reagents and methods available for any given test, it is unlikely they will all perform these test in the same way. This variability will compromise patient management because different test results can arise from testing the same sample. As test result will typically direct further clinical management, different data may lead to different clinical actions.

Moreover, even using the same test reagents and instruments can still lead to differences in test results. 


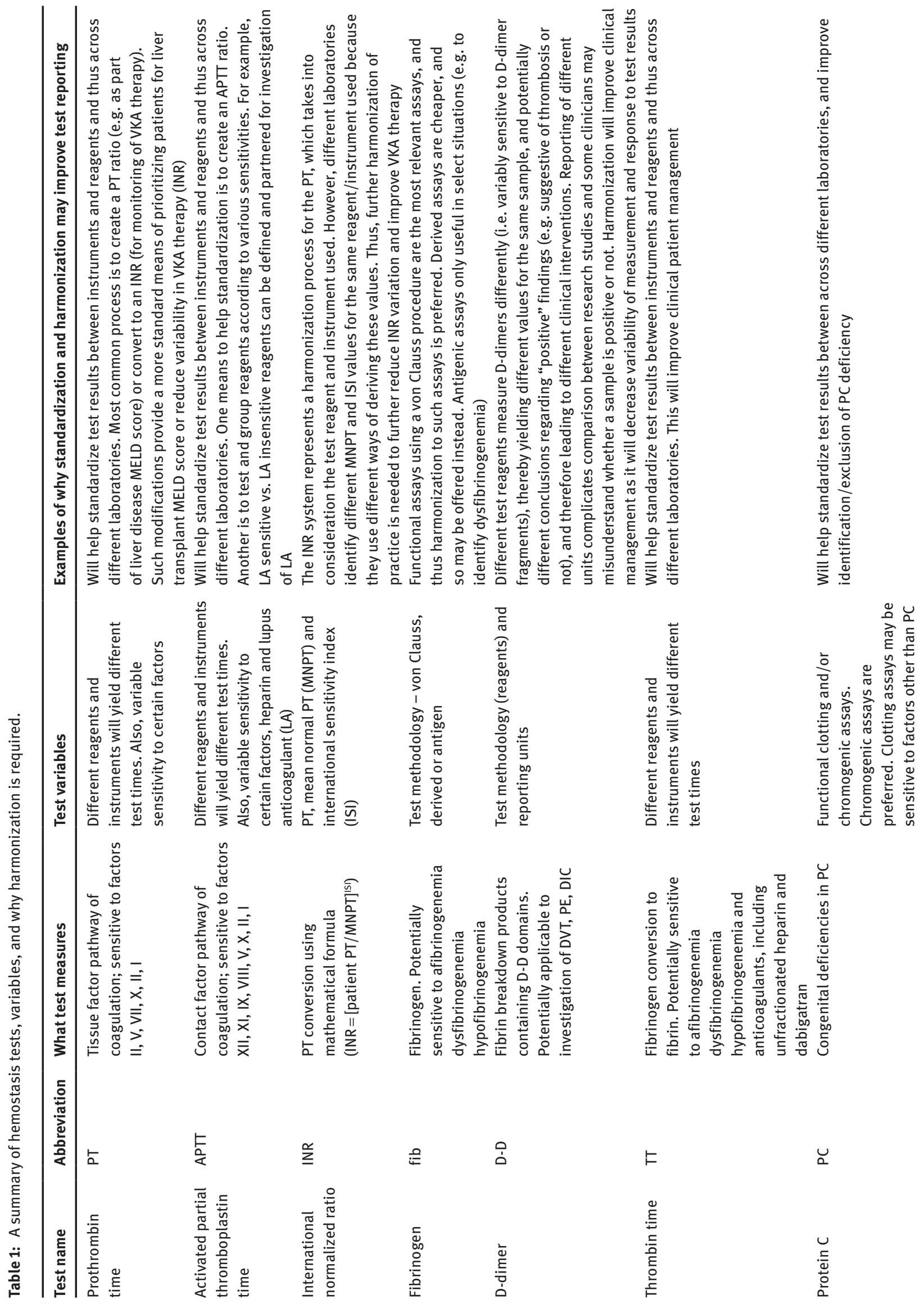




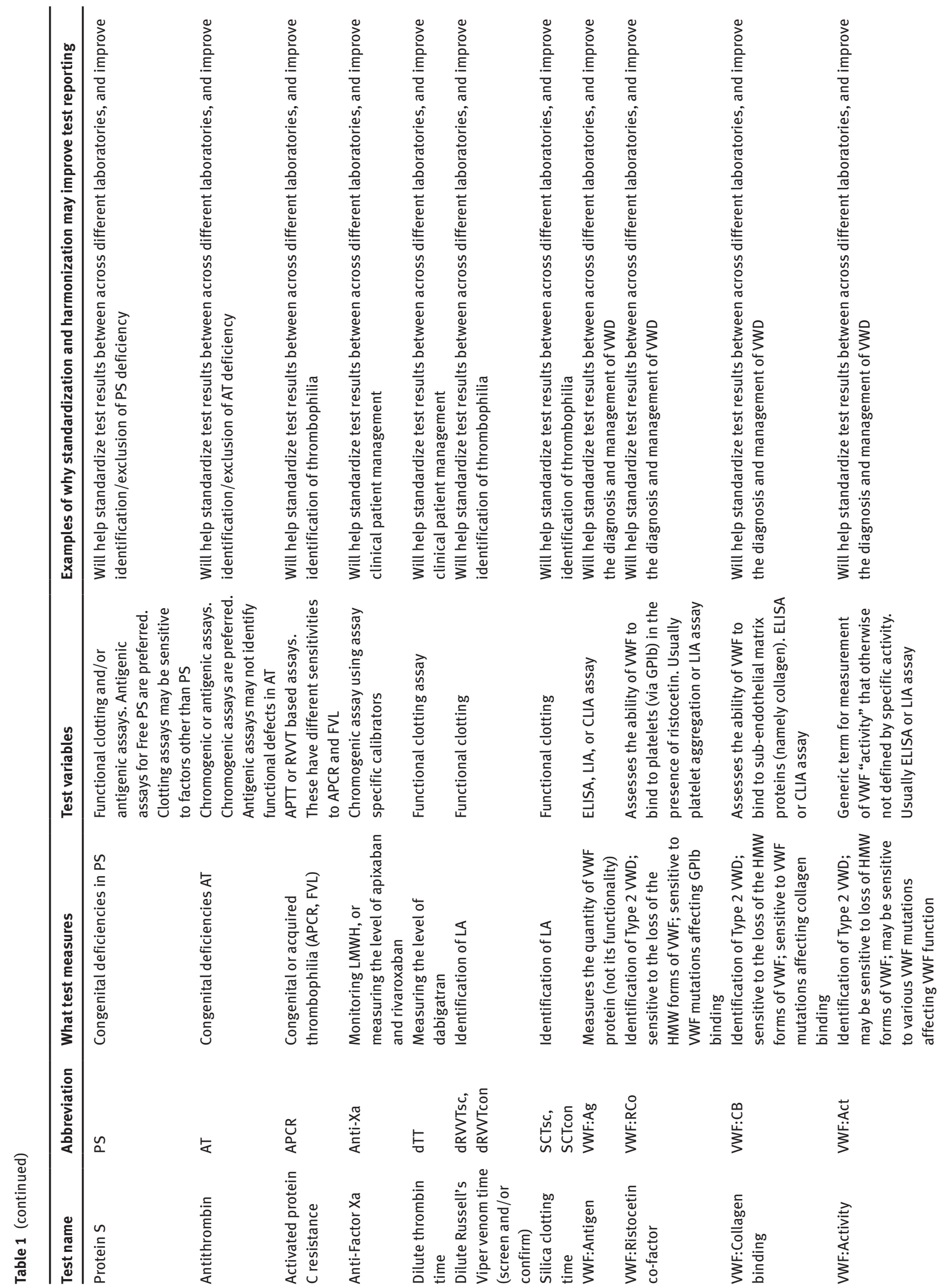




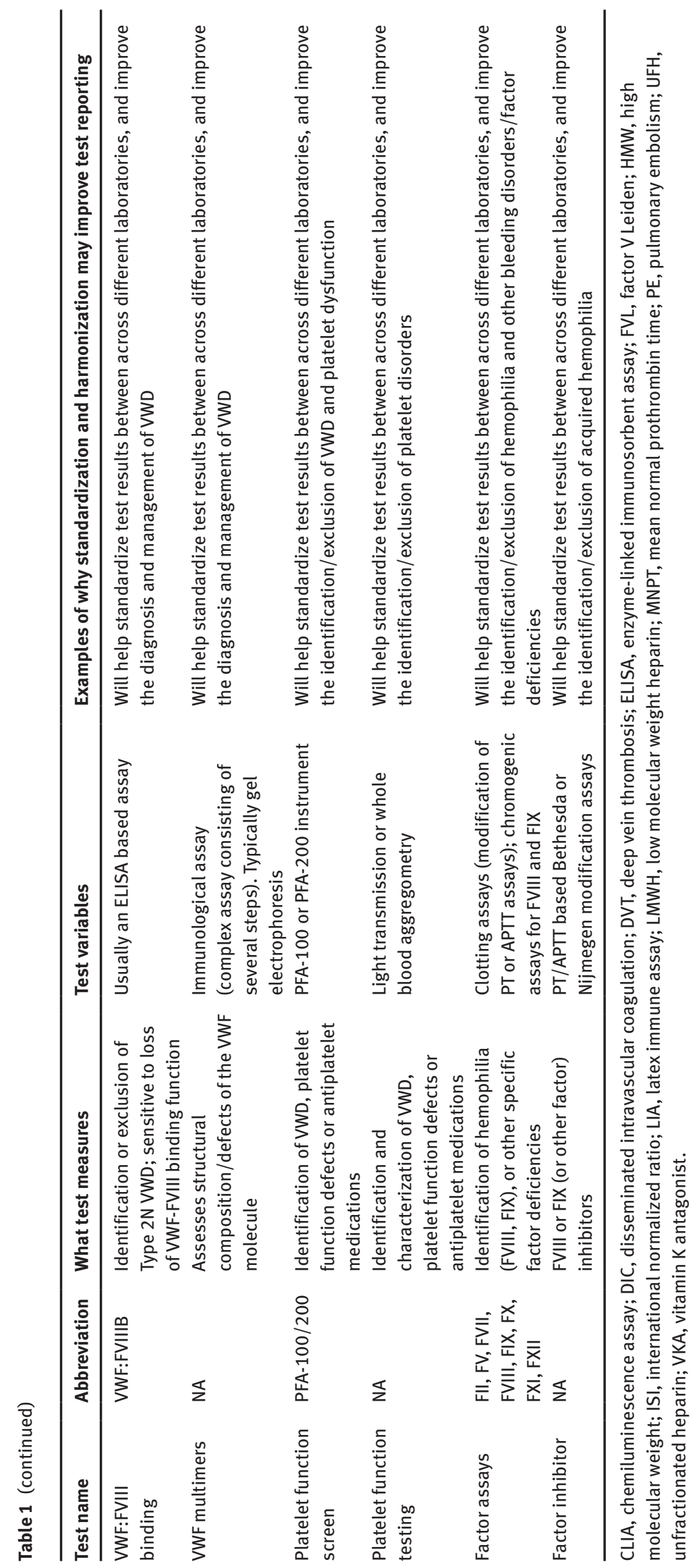


As a pragmatic example, consider the routine tests "prothrombin time" (PT) and "activated partial thromboplastin time" (APTT). The PT is used for several reasons, including evaluation of the tissue factor pathway of coagulation, for prioritizing liver transplantation in end stage liver disease (usually as a PT ratio of patient PT/normal PT) and for monitoring patients on VKA therapy (usually as an international normalized ratio [INR]) (Table 1). The APTT is also used for several reasons, including evaluation of the contact factor pathway of coagulation, as a screen for hemophilia, for identification of lupus anticoagulant (LA; a prothrombotic disorder) and for monitoring of anticoagulant therapy with unfractionated heparin. First, different reagents and different instruments, and the arising various combinations, can yield vastly different PT and APTT values among laboratories. Second, although normalizing test values by use of test ratios (as a form of test harmonization) or producing an INR will help reduce this variability, it will not completely eliminate it. Indeed, laboratories using the same instrument and the same test reagent (even the same lot of reagent) may still generate highly disparate INR values, simply because they have calculated the components of the INR equation (namely, mean normal prothrombin time [MNPT] and international sensitivity index [ISI]) in different ways [5, 6]. For patients on VKA therapy, the recommended INR is usually around 2.03.0 for most indications. In some cases, as identified in external quality assessment (EQA) surveys, different laboratories can yield differences in INR that can range from 2.4 to over 6.0 for the sample homogenous sample [5], as also shown in Figure 1. A value of 2.4 would be considered therapeutic, and no clinical intervention would be needed (the patient would likely continue the same dose of VKA until next testing). However, an INR value of 6 is typically considered an alert (critical) value [7] and would suggest clinical intervention - reducing dosage and possibly close monitoring for potential bleed events. Although the wide range of reported INRs is influenced by outlier INRs, most INRs fall in the region of 3.3-4.5 for this example, which may still represent different clinical responses. As explanation for the wide INR range, it can be reflected that participant reported ISI and MNPT values were also widely variable and could have theoretically led to the wide INR values, as highlighted in Figure 1. Similarly, for the APTT, a heparin therapeutic range can be exceeded or not for the same sample as tested in different laboratories [8]. Moreover, the new direct oral anticoagulants may also (variably) affect routine coagulation tests such as PT and APTT, and/or these tests may be used to help identify the presence or absence of such drugs $[9,10]$.

As another example, D-dimer testing is plagued not only by differences in test reagents and methods, leading to differences in test results for the same sample, but also by inconsistency in reporting units [11, 12]. In fact, 28 different combinations of units are feasible, thereby acting to considerably confuse clinicians who order these tests and thereafter manage patients. Notably then, based on differing reagents, the same sample may be identified as "positive" or "negative" for D-dimer based on different test values. However, even with the same reagent,

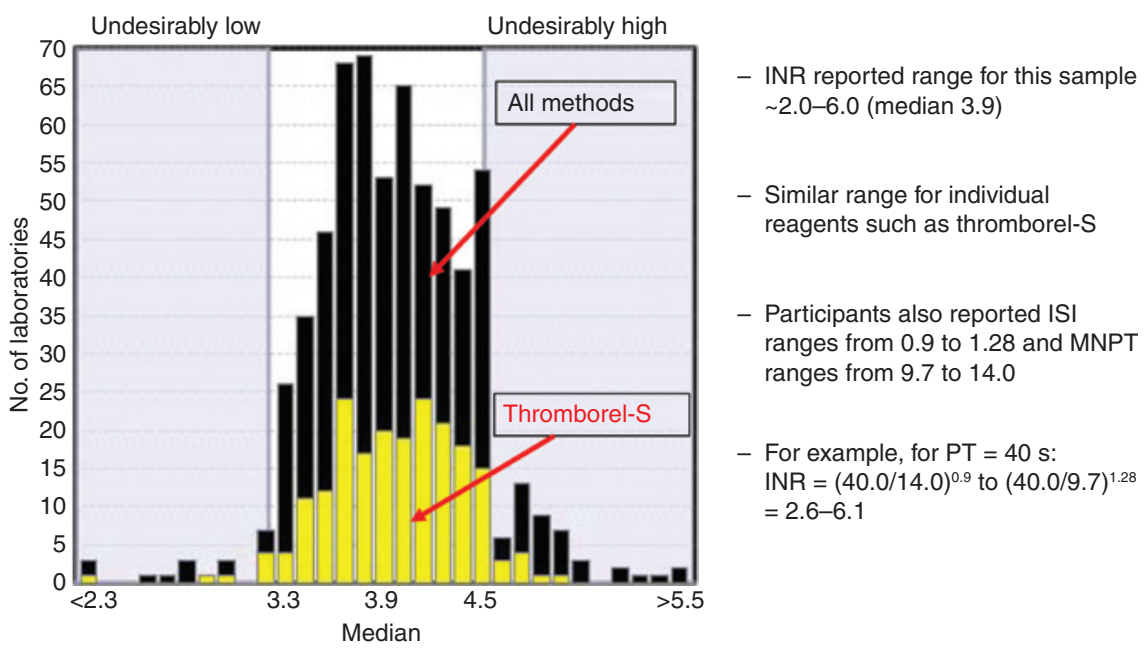

Figure 1: An example of external quality assessment (EQA) for an individual sample as assessed in a local EQA program in Australia (the RCPAQAP Hematology [7]).

A wide range of INR values was reported by laboratories for the same homogeneous sample. 
the same sample may also be interpreted as "positive" or "negative" by different clinicians for the same numerical value, depending on their experience/inexperience with the reported unit [13].

Another final example is VWF testing for VWD diagnosis/exclusion. VWF is a large and complex protein with multiple functionalities, including binding to platelets, subendothelial matrix components such as collagen, and binding to FVIII (thereby protecting it from degradation and also delivering it to sites of injury). VWD can arise from defects in any of these activities. Thus, identification/ exclusion/characterization of VWD requires the use of multiple assays [14]. This may not seem a problem because indeed an arsenal of potential assays exist in laboratories (Table 1). However, each of these assays measures different aspects of VWF (level/different activities), and laboratories may use many different combinations of assays as test panels or may not understand strengths and limitations of the assays they use individually or in composite, thereby leading to many (potentially avoidable) errors in VWD diagnosis [15]. Moreover, these same assays may be used to monitor therapy in VWD, with the same limitations applied in terms of current knowledge, and corresponding adverse outcomes in regards to differential therapies $[4,16]$.

Standardization and harmonization is intended to reduce the variability of laboratory test practice and, thus, to provide more consistent standards of health care. In this issue of the journal, there are several papers describing various initiatives that attempt to drive standardization and harmonization of hemostasis test practice at local, regional or international levels $[17,18]$.

Hemostasis is complex. Standardization and harmonization aims to make understanding hemostasis less complex and tests of hemostasis more useful. We hope readers enjoy this issue of the journal, and in particular, the papers related to harmonization of hemostasis test practice.

Author contributions: EJF wrote the original manuscript. Both coauthors contributed to manuscript revision and have approved the final manuscript for publication. All the authors have accepted responsibility for the entire content of this submitted manuscript and approved submission.

Research funding: None declared.

Employment or leadership: None declared.

Honorarium: None declared.

Disclosure: The authors state that they have no interest that might be perceived as posing a conflict or bias.

\section{References}

1. Bonar RA, Lippi G, Favaloro EJ. Overview of hemostasis and thrombosis and contribution of laboratory testing to diagnosis and management of hemostasis and thrombosis disorders. Methods Mol Biol 2017;1646:3-27.

2. Lippi G, Favaloro EJ. Laboratory hemostasis: from biology to the bench. Clin Chem Lab Med 2018 Feb 19; doi: 10.1515/cclm-20171205. [Epub ahead of print].

3. Lippi G, Franchini M, Favaloro EJ. Diagnostics of inherited bleeding disorders of secondary hemostasis: an easy guide for routine clinical laboratories. Semin Thromb Hemost 2016;42:471-7.

4. Curnow J, Pasalic L, Favaloro EJ. Treatment of von Willebrand disease. Semin Thromb Hemost 2016;42:133-46.

5. Bonar R, Favaloro EJ. Explaining and reducing the variation in inter-laboratory reported values for International Normalised Ratio. Thromb Res 2017;150:22-9.

6. Tripodi A, Lippi G, Plebani M. How to report results of prothrombin and activated partial thromboplastin times. Clin Chem Lab Med 2016;54:215-2.

7. Lippi G, Adcock D, Simundic AM, Tripodi A, Favaloro EJ. Critical laboratory values in hemostasis: toward consensus. Ann Med 2017;49:455-61.

8. Baluwala I, Favaloro EJ, Pasalic L. Therapeutic monitoring of unfractionated heparin - trials and tribulations. Expert Rev Hematol 2017;10:595-605.

9. Favaloro EJ, Pasalic L, Curnow J, Lippi G. Laboratory monitoring or measurement of direct oral anticoagulants (DOACs): advantages, limitations and future challenges. Curr Drug Metab 2017;18:598-608.

10. Favaloro EJ, Pasalic L, Lippi G. Replacing warfarin therapy with the newer direct oral anticoagulants, or simply a growth in anticoagulation therapy? Implications for Pathology testing. Pathology 2017;49:639-43.

11. Thachil J, Lippi G, Favaloro EJ. D-Dimer testing: laboratory aspects and current issues. Methods Mol Biol 2017;1646:91-104.

12. Longstaff C, Adcock D, Olson JD, Jennings I, Kitchen S, Mutch N, et al. Harmonisation of D-dimer - A call for action. Thromb Res 2016;137:219-20.

13. Lippi G, Tripodi A, Simundic AM, Favaloro EJ. International survey on D-dimer test reporting: a call for standardization. Semin Thromb Hemost 2015;41:287-93.

14. Favaloro EJ, Pasalic L, Curnow J. Laboratory tests used to help diagnose von Willebrand disease: an update. Pathology 2016;48:303-18.

15. Favaloro EJ, Bonar RA, Meiring M, Duncan E, Mohammed S, Sioufi J, et al. Evaluating errors in the laboratory identification of von Willebrand disease in the real world. Thromb Res 2014;134:393-403.

16. Favaloro EJ, Pasalic L, Curnow J. Monitoring therapy during treatment of von Willebrand disease. Semin Thromb Hemost 2017;43:338-54.

17. Favaloro EJ, Jennings I, Olson J, Van Cott EM, Bonar R, Gosselin $\mathrm{R}$, et al. Towards harmonization of external quality assessment/ proficiency testing in hemostasis. Clin Chem Lab Med 2018.

18. Favaloro EJ, Gosselin R, Olson J, Jennings I, Lippi G. Recent initiatives in harmonization of hemostasis practice. Clin Chem Lab Med 2018;56:1608-19. 
Corresponding author: Dr. Emmanuel J. Favaloro, Department of Haematology, Institute of Clinical Pathology and Medical Research (ICPMR), NSW Health Pathology, Westmead Hospital, Westmead, NSW, 2145, Australia; and Sydney Centres for Thrombosis and Haemostasis, Westmead, NSW, Australia,
Phone: +(612) 8890 6618, Fax: +(612) 9689 2331,

E-mail: emmanuel.favaloro@health.nsw.gov.au

Giuseppe Lippi: Section of Clinical Biochemistry, Department of Neurological, Biomedical and Movement Sciences, University of Verona, Verona, Italy. http://orcid.org/0000-0001-9523-9054 